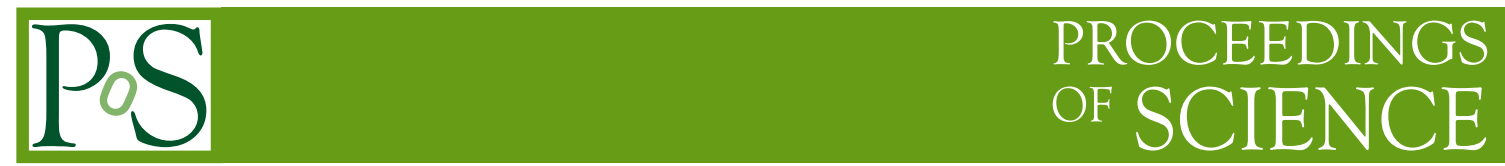

\title{
New half-life results on very long-living nuclei
}

\author{
Kai Zuber*i \\ Institute for Nuclear and Particle Physics, TU Dresden, Germany \\ E-mail: zuber@physik.tu-dresden.de
}

\begin{abstract}
New half-live measurements for the $\alpha$-decays of ${ }^{147} \mathrm{Sm}$ and ${ }^{190} \mathrm{Pt}$ are given. Improved half-lives and limits of highly forbidden beta decays have been obtained for ${ }^{50} \mathrm{~V}$ and ${ }^{180 m} \mathrm{Ta}$. Furthermore, new half-life limits for various forms of double electron capture are presented.
\end{abstract}

The 26th International Nuclear Physics Conference

11-16 September, 2016

Adelaide, Australia

\footnotetext{
* Speaker.

$\dagger$ I want to thank all my collaborators helping with these measurements, especially B. Lehnert, H. Wilsenach, T. Wester and D. Degering
} 


\section{New results on $\alpha$-decay half-lives}

The study of alpha decays has been important for the understanding of nuclei and their properties for more than a century. Currently these studies still have impact in various areas of nuclear physics, providing information which is valuable and often not accessible otherwise. The half-lives are sensitive to nuclear structure and the half-life region between $10^{6-10}$ years and beyond is very interesting as several nuclides in this range are used as (cosmo-)chronometers and the limitations on dating precision might be restricted by the accuracy in the known half-life. Furthermore, scanning through the available data there is a reasonable spread in half-life measurements for individual nuclei, which might deserve better precision.

To study $\alpha$-decays experimentally a new Frisch-grid ionisation chamber was built made out of low

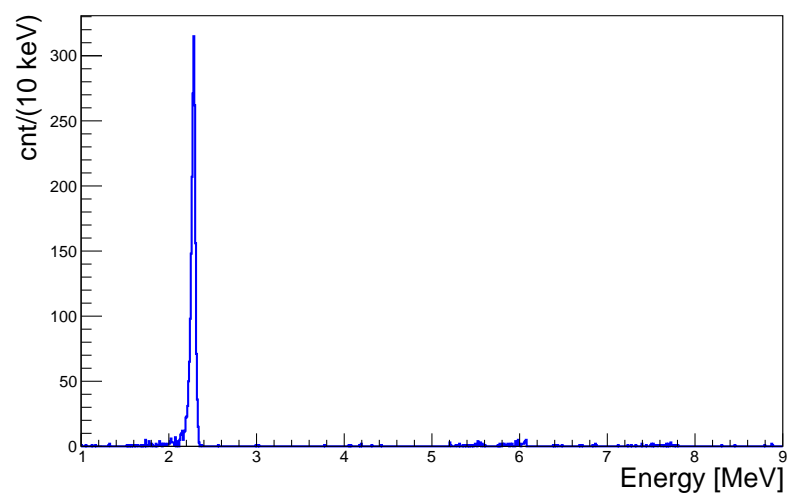

Figure 1: Peak of ${ }^{147} \mathrm{Sm}$ including background. The peak is clearly visible and a high signal to background ratio is achieved.

level radioactive materials [1]. This is due to the fact that major interest is in long-living nuclides, of course the chamber can be used for any $\alpha$-decay. Following the Geiger-Nuttall law, long halflives lead to lower Q-values, typically below $3 \mathrm{MeV}$. In a background run of 30.8 days it could be shown that the chamber has a background in the region of 1-3 MeV of only one event per 2 days. For more details on the chamber see [1]. In a first attempt a measurement of the ${ }^{147} \mathrm{Sm}$ half-life was envisaged. Even so this is a classical calibration source the measured values over time still show some scattering. In a new measurement with very thin targets more than 60000 events were collected, a fraction of the data is shown in Fig. 1. The half-live obtained is [2]

$$
\left.T_{1 / 2}=(1.0803 \pm 0.0099 \text { (stat. }) \pm 0.0241(\text { (sys. })\right) \times 10^{11} \text { years }
$$

in very good agreement with the most precise value claimed by [3]. In addition, also the half-life into the first excited state of ${ }^{143} \mathrm{Nd}$ has been explored using low background gamma-spectroscopy and results in $T_{1 / 2}>3.3 \times 10^{18} \mathrm{yrs}(90 \% \mathrm{CL})$. Such an excited state search was also performed for the ${ }^{146} \mathrm{Nd}$ decay into the first excited state of ${ }^{142} \mathrm{Ce}$. Here, the lower half-live limit obtained was $T_{1 / 2}>1.6 \times 10^{18} \mathrm{yrs}(90 \% \mathrm{CL})[4]$.

In a next step the $\alpha$-decay half-live of ${ }^{190} \mathrm{Pt}$ has been explored. Here there is a significant spread between laboratory measurements, cosmochronological results and semi-empirical fitting of $\alpha$ emitters. Previous laboratory measurements have uncertainties of at least $10 \%$, while geological samples are presented with errors less than $1 \%$ (for a compilation of results see [5]). The new 
measurement (Fig. 2) resulted a half life of

$$
T_{1 / 2}=(4.97 \pm 0.16) \times 10^{11} \text { years }
$$

with statistical and systematic errors added in quadrature [6]. This is the most precise laboratory measurement and agrees well with the value extracted from cosmochronological data.

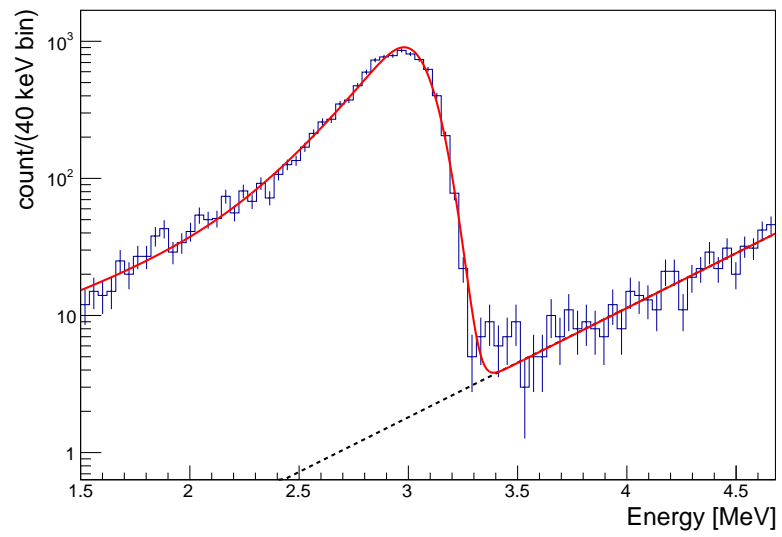

Figure 2: Logarithmic presentation of the peak of ${ }^{190} \mathrm{Pt}$ including background. The latter can be considered to be very small.

\section{Study of highly forbidden beta decays}

Highly forbidden beta decays $(\Delta I>2)$ are interesting by themselves as the log ft-values are well above 10 and the spectral shapes are extremely difficult to calculate. One example is ${ }^{50} \mathrm{~V}$ with its ground state of $6^{+}$. It has two decay branches into a $2^{+}$state of ${ }^{50} \mathrm{Ti}$ and ${ }^{50} \mathrm{Cr}$. These decays are searched for more than 6 decades, with several evidences which were later replaced by more stringent lower limits. Finally, the EC into the Ti-branch has been observed with good statistics to be

$$
T_{1 / 2}=(2.28 \pm 0.25) \times 10^{17} \text { years }
$$

while the beta-decay into the $\mathrm{Cr}-50$ branch resulted in a lower limit of $T_{1 / 2}>1.5 \times 10^{18}$ years [8]. This triggered some shell model calculations to predict the missing half-live. It turns out that this is expected to be an order of magnitude higher than the current experimental limit [9].

Another example is ${ }^{180 m} \mathrm{Ta}$, often called nature's rarest isotope. The isomeric $9^{+}$state serves as ground state and can in principle decay into two different $6^{+}$states in a similar fashion as the previous nuclide. A new search has been performed using Bayesian statistics and is improving the lower limits of the half-life to $T_{1 / 2}>5.8 \times 10^{16}$ years for the beta-decay branch and $T_{1 / 2}>$ $2.0 \times 10^{17}$ years for the electron-capture branch respectively [7]. Combining these two values results in an overall half-life limit of $T_{1 / 2}>4.5 \times 10^{16}$ years.

\section{Very rare event searches- Double electron capture}

A very important search is double beta decay, especially the neutrino-less mode. While this is massively investigated there is an alternative process providing additionally information namle 
double positron decay, double electron capture (EC) and the mixed mode. These three different decay modes can be considered:

$$
\begin{array}{rr}
(Z, A) \rightarrow(Z-2, A)+2 e^{+}+\left(2 v_{e}\right) & \left(\beta^{+} \beta^{+}\right) \\
e^{-}+(Z, A) \rightarrow(Z-2, A)+e^{+}+\left(2 v_{e}\right) & \left(\beta^{+} / \mathrm{EC}\right) \\
2 e^{-}+(Z, A) \rightarrow(Z-2, A)+\left(2 v_{e}\right) & (\mathrm{EC} / \mathrm{EC})
\end{array}
$$

From phase space arguments the double EC should be most likely and its 2 neutrino half-life should be in the same range as for the corresponding double beta decays. Another interesting aspect is neutrino-less double EC. This would violate momentum conservation, hence the easiest way considered to solve this issue is to emit a photon, hence called radiative double EC. Various new measurements have been performed in the past, a few are mentioned here [10, 11, 12, 13]. A half-life limit of $T_{1 / 2}>4.0 \times 10^{21}$ years is given for the ${ }^{110} \mathrm{Pd}$ double beta decay into the first excited state [10]. The radiative double $\mathrm{EC}$ of ${ }^{36} \mathrm{Ar}$ is constrained to be larger than $T_{1 / 2}>3.6 \times 10^{21}$ years [13] and the same mode for ${ }^{58} \mathrm{Ni}$ to $T_{1 / 2}>2.1 \times 10^{21}$ years [12].

\section{References}

[1] A. Hartmann et al., Nucl. Instrum. Methods A 814, 1 (2016)

[2] D. Degering et al., submitted

[3] K. Kossert et al., Appl. Rad. Isot. 67, 1702 (2009)

[4] C. Stengl, H. Wilsenach, K. Zuber Int. J. Mod. Phys. E 24, 1550043 (2015)

[5] O. Tavares et al., Nucl. Instrum. Methods B 243, 256 (2006)

[6] B. Mihaly et al., submitted

[7] B. Lehnert et al., arXiv:1609.03725

[8] H. Dombrowski, S. Neumaier, K. Zuber Phys. Rev. C 83, 0865201 (2011)

[9] M. Haaranen, P. C. Srivastava, J. Suhonen, K. Zuber, Phys. Rev. C 90, 044314 (2014)

[10] B. Lehnert et al., Journal of Physics G 43, 054322 (2016)

[11] B. Lehnert et al., Journal of Physics G 43, 085201 (2016)

[12] B. Lehnert et al., Journal of Physics G 43, 065201 (2016)

[13] M. Agostini et al., Europ. Phys. J. C 76, 652 (2016) 